\title{
Lightweight route adjustment strategy for mobile sink wireless sensor networks
}

\author{
Ahmed Basil Ghazi ${ }^{1}$, Omar Adil Mahdi², Wid Badee Abdulaziz ${ }^{3}$ \\ ${ }^{1,2}$ Department of Computer Sciences, College of Education for Pure Sciences-Ibn Al-Haytham, University of Baghdad, \\ Baghdad, Iraq \\ ${ }^{3}$ University of Information Technology and Communications, Baghdad, Iraq
}

\begin{tabular}{l}
\hline Article Info \\
\hline Article history: \\
Received Apr 12020 \\
Revised Jul 15, 2020 \\
Accepted Jul 28, 2020 \\
\hline
\end{tabular}

\section{Keywords:}

Energy consumption

Mobile sink

Overhead

Route adjustment

Wireless sensor networks

\begin{abstract}
As a result of numerous applications and low installation costs, wireless sensor networks (WSNs) have expanded excessively. The main concern in the WSN environment is to lower energy consumption amidst nodes while preserving an acceptable level of service quality. Using multi-mobile sinks to reduce the nodes' energy consumption have been considered as an efficient strategy. In such networks, the dynamic network topology created by the sinks mobility makes it a challenging task to deliver the data to the sinks. Thus, in order to provide efficient data dissemination, the sensor nodes will have to readjust the routes to the current position of the mobile sinks. The route re-adjustment process could result in a significant maximization in the communication cost, which boosts the total energy depletion. This paper proposes a lightweight routes re-adjustment strategy for mobile sink wireless sensor networks (LRAS-MS) aimed at minimizing communication cost and energy consumption by reducing route re-adjustment in a cluster-based WSN environment. The simulation results show a significant reduction in communication costs and extending the network lifetime while maintaining comparable low data delivery delay.
\end{abstract}

This is an open access article under the CC BY-SA license.

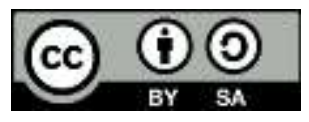

\section{Corresponding Author:}

Omar Adil Mahdi

Department of Computer Sciences

College of Education for Pure Sciences-Ibn Al-Haytham

University of Baghdad, 10071 Baghdad, Iraq

Email: omar.a.m@ihcoedu.uobaghdad.edu.iq

\section{INTRODUCTION}

The current progress in wireless communication technology has made the microelectronics devices smaller and cheaper, making a huge number of wireless sensors economically reasonable. However, even with limitations a group of sensors together can build an effective wireless sensor network (WSN) for operations requiring durable operations. These facts introduced the WSNs as an up-and-coming technology together with numerous different applications, such as structural health monitoring [1], intrusion detection [2], border surveillance [3], activity recognition [4], intelligent transportation [5], disaster control [6], unapproachable places monitoring [7], and etc. The primary objective of the WSN is to gather information by observing the surrounding environment and detect any unusual events or observing some environmental variables. To accomplish these tasks, each sensor in the WSN must independently sense the local environment, yet achieve a collective gathering of complex information. Where sensor-source nodes produce information on a phenomenon and send data streams to a richer resource device termed a sink [8, 9], a WSN generally supports the converge cast communication model. All the sensors in the traditional WSN system 
forward the gathered data into a common static sink, and it can become a problem in the long term [10]. Specifically, with restricted energy supply to network nodes, it causes WSNs to deal with critical functional restrictions $[11,12]$. Deploying a static sink would shorten the lifetime of the sink's neighbors due to the gradual depletion of energy nodes and cause them to die earlier than other nodes [13, 14]. Therefore, using multiple sinks instead of one sink can spread the load over the network and this can be viewed as a good way to reduce the energy problem for a while, but still not so efficient $[15,16]$. Thus, using mobile sinks rather than static one can prevent exceeding transmission on nodes nearby the position of the static sinks and maximize a lifetime of the network, such a network is called a mobile sink-based Wireless Sensor Networks (mWSN) [17]. While employing sink mobility results in a considerable improvements in the network performance, it additionally creates new issues for the dissemination of data in WSNs that the routing protocols need to address [18]. Therein, due to the dynamic topology of the network in time, each routing protocol that works with sink mobility must perform the extra operations [19]. The most prominent operation that can cause an increase in energy consumption is the need for sensor nodes to constantly preserve the mobile sink's new position to report sensed data of the field being monitored. Even though various data transmission protocols to sensor networks were suggested, most of them imply that any mobile sink should stream its position information regularly via the sensor field. This way every sensor is informed about the position of the sink for next measurements to be sent. Nonetheless, this strategy leads to raising overhead in wireless network especially with the multi-mobile sinks.

This paper will present a lightweight routes readjustment strategy for wireless sensor networks based on mobile sinks (LRAS-MS) that construct a uniform clusters on the monitoring field. From every cluster, the proposed strategy will elect a cluster-head $(\mathrm{CH})$. The $\mathrm{CH}$ main job is to gathering observation data from the clusters' member nodes and retransmit it toward the mobile sink. The proposed strategy aims to reduce the routes readjustment cost in to the multi-mobile sinks WSN by involving only a sub-group of $\mathrm{CHs}$ in routes readjusting process. Moreover, LRAS-MS discovering the nearly closest mobile sink to the nods with optimum routes to the most recent location of the mobile sink with a least overhead network.

\section{RELATED WORKS}

Data dissemination protocols based on mobile sink-based wireless sensor networks (mWSNs) have been suggested over the past decade. There are two types of data collection or dissemination strategy: controlled and uncontrolled sink mobility strategies. They are dependent on the mobility model displayed by the sink in the monitoring area. The sink's speed and/or direction (sink mobility) in controlled sink mobility strategies is employed and regulated by an external observer [20-22]. Uncontrolled sink-based mobility strategies are defined by the fact that, as to mobility, the sink forms its next step independently [23]. We define the related works about controlled sink mobility, containing not only the methodology but also comparative strengths and weaknesses. Moreover, the important features, overhead control mechanism and design goals of the proposed data dissemination protocol in the literature are described in Table 1.

The anchor-based voronoi-scoping routing protocol (AVRP) [24] focuses on minimizing overhead and boosting data transmission performance to adapt to a mobile sink. The authors have improved VoronoiScoping which combines the anchor nodes with the mobile sink. AVRP sets up a delivery structure and regularly refreshes the structure based on the sinks ' movement. Thus, all sensors only need to store the routing information of the closest anchor node, and AVRP eliminates the need for information of the dynamic routing path, which is a significant data overhead in transmission. Nevertheless, additional control packets have to be streamed inside the Voronoi domain of mobile sinks at any time a sink moves.

An effective data-driven routing protocol (DDRP) for minimizing the control overhead of network in route maintenance and improving the performance of data delivery rate was suggested by Shi et al. [25]. According to this protocol, the mobile sink uses the wireless medium's broadcast characteristic and systematically transmits beacon messages with a timestamp field, sink ID field, and beacon-interval variable (optional field) to its single hop neighbors as they move around. The neighbor sensor nodes thus being aware to sink's presence in their surrounding zone. The major disadvantage of DDRP approach is adopted overhearing scenario to follow the current position of the sink will enforce the nodes to be in listening mode, which is significantly depleting the energy reserves of nodes.

Multiple enhanced specified-deployed sub-sinks (MESS) [26] create a virtual band in the center of the nodes area, thus putting improved nodes referred to as sub-sinks with better storage space at equivalent dimensions. The group of sub-sink nodes over the available path serves as mobile sink meeting points, gathers, and stores sensed data from different nodes. In MESS approach the mobile sink floods the request on the virtual strip in the data delivery stage until it meets the data-owned sub-sink node. The sub-sinks direct the collected information to the mobile sink using a geographical delivering method once receiving the request from the mobile sink. 
The Virtual grid-based dynamic routes adjustment scheme (VGDRA) [27] introduced a way to develop a virtual infrastructure and conduct valid routes to the recent update of the mobile sink location. The VGDRA scheme separates the monitored region into a virtual grid of uniform cells, building a virtual network. A collection of nodes near the midst of the cells are called cell headers. They trace the mobile sink's current location and mitigate the cell member nodes from engaging in the re-adjustment process of the routes. The cell member nodes interact with the nearest cell headers and message them the collected data while the ajacent cell headers interact via gateway nodes with each other.

Table 1. Comparison of different sink mobility based data routing protocols for mWSN

\begin{tabular}{ccccccc}
\hline Variable & $\begin{array}{c}\text { Location- } \\
\text { based }\end{array}$ & $\begin{array}{c}\text { Sink mobility } \\
\text { constrain }\end{array}$ & $\begin{array}{c}\text { Reporting } \\
\text { Mode }\end{array}$ & $\begin{array}{c}\text { No. of } \\
\text { Sinks }\end{array}$ & Overhead Control Mechanism \\
\hline AVRP & No & Constant speed & Proactive & Multiple & $\begin{array}{c}\text { Limited by each mobile sink's } \\
\text { Voronoi scope }\end{array}$ & $\begin{array}{c}\text { Improve the performance of } \\
\text { data delivery }\end{array}$ \\
MDRP & No & No constraints & Periodic & Multiple & $\begin{array}{c}\text { Mechanism for overhearing } \\
\text { Extending the life of the } \\
\text { network }\end{array}$ & $\begin{array}{c}\text { Long-distance communication } \\
\text { between the nodes of the } \\
\text { source and the sink }\end{array}$ \\
VGDRA & No & No constraints & Proactive & Single & $\begin{array}{c}\text { Exploit multiple static sub- } \\
\text { sinks }\end{array}$ & $\begin{array}{c}\text { Minimize the routes } \\
\text { reconstruction cost }\end{array}$ \\
\hline
\end{tabular}

\section{DESCRIPTION OF LRAS-MS STRATEGY}

The main objective of the proposed LRAS-MS strategy is to reduce the cost of communication in sensor networks by eliminating from route adjustment process the unnecessary sensor nodes. This section provides an overview of the proposed strategy, this detailed description involves the initialization stage; construction of the backbone route structure and the formation of clusters together with the election of the heads. While the re-adjustment stage; explains how to maintain the new routes to the mobile sinks when the locations of the sinks are changed.

\subsection{Initialization}

In the proposed strategy, the initialization stage is done immediately after the nodes are deployed. The LRAS-MS strategy generates a cluster of sensor nodes of equal size. A single node is selected to be a cluster-head $(\mathrm{CH})$ from each cluster. Whereas the other cluster nodes simultaneously set their rules to clustermembers (CM). The duty of each cluster head is to gathering the consecutive observed data from the member nodes and redirected it to the nearest mobile sink. The mobile sinks take positions at the coordinates $(0,0)$ and $(n, n)$ of the monitoring field, considering the field dimension size is $(n \times n)$. Mobile sinks move counterclockwise around the sensor field and transmit the route establishment message (REM) to the nearest cluster heads on a regular basis. The REM header comprises four main parameters, namely Type (packet type), Sink-ID (the sink ID that transmitted the REM), CH-ID (the cluster-head node ID that transmitted the REM), and hop2sink (the hop distance to the sink). The structure and roles that are used in the routing arrangement of LRAS-MS are illustrated in Figure 1.

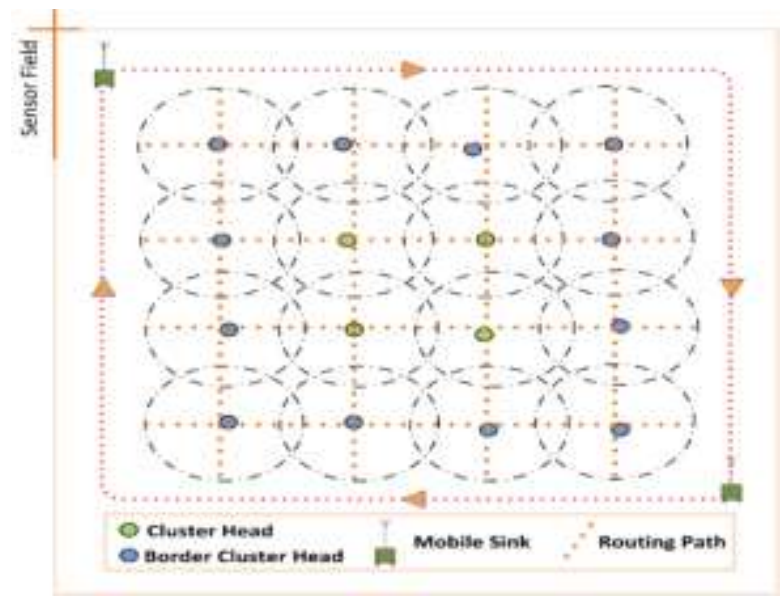

Figure 1. Network model diagram of LRAS-MS 
The REM is utilized in the LRAS-MS strategy to inform the cluster-heads with the sinks' position, these cluster-heads will update the sink-hop by increasing its value and retransmit the message to its $\mathrm{CH}$ neighbors. When a cluster-head receive more than one REM message it will compare the values of hop $2 \operatorname{sink}$ included in a message and consider the smallest hop2sink value as a preferred route.

\subsection{Routes re-adjustment}

The data delivery routes of the cluster-heads (CHs) need to be set up according to the mobile sinks' latest location. Just a few CHs maintain new routes to the current mobile sink position using our LRAS-MS strategy. The mobile sinks move around the sensor field, gathering information through the $\mathrm{CH}$ of the closest borderline. Just a few CHs engage in the LRAS-MS strategy in the process of re-adjusting routes that require changing their routes. However, the mobile sink when changes its position, a new REM (with hop2sink equal 0 ) will be transmitting to the closest border-line cluster-head. Once the REM message received by border $\mathrm{CH}$, the $\mathrm{CH}$ check whether or not the next-hop is on the mobile sink. Then, regulated by the previous condition validation, the border $\mathrm{CH}$ does not transmit the obtained REM, which means that the $\mathrm{CH}$ next hop is the mobile sink. On the contrary, it means the border $\mathrm{CH}$ set the ID of the mobile sink which, included in received REM as its next hop. Furthermore, the received REM message will be updated by increasing the hop2sink value, update the $\mathrm{CH}$-ID field and then retransmit the message to the neighbor $\mathrm{CHs}$. Upon receiving the REM from the neighbor $\mathrm{CHs}$, it will check if $\mathrm{CH}$-ID in a received REM is equal to its next hope. If it is the same, the $\mathrm{CH}$ drops the REM; therefore, initiating a different route as the new backbone structure is unnecessary. Otherwise, the $\mathrm{CH}$ updates its next-hop to the sender of REM. Later, the $\mathrm{CH}$ update and retransmitted the REM to its neighbors. In a case the $\mathrm{CH}$ received more than one REM at the same time, the message with the lowest hop2sink value will be opted as a preferred route. Figure 2 shows the flowchart of routes re-adjustment procedure.

The LRAS-MS strategy tracks the current cluster head's energy level, where a sensitive energy threshold is utilized to specify the $\mathrm{CH}$ rotation procedure. The threshold is calculated on the basis of the $\mathrm{CH}$ residual energy (Eres) as $(\mathrm{P} \times$ Eres), where $\mathrm{P}$ is a constant parameter ranged from 0 to 1 . The new cluster head election is only initiate if the residual energy of the current $\mathrm{CH}$ falls below or equal the threshold value. In case of $\mathrm{CH}$ rotation, the new $\mathrm{CH}$ shares its information with the cluster head of neighbors and with the nodes of its cluster to maintain the backbone structure of the network.

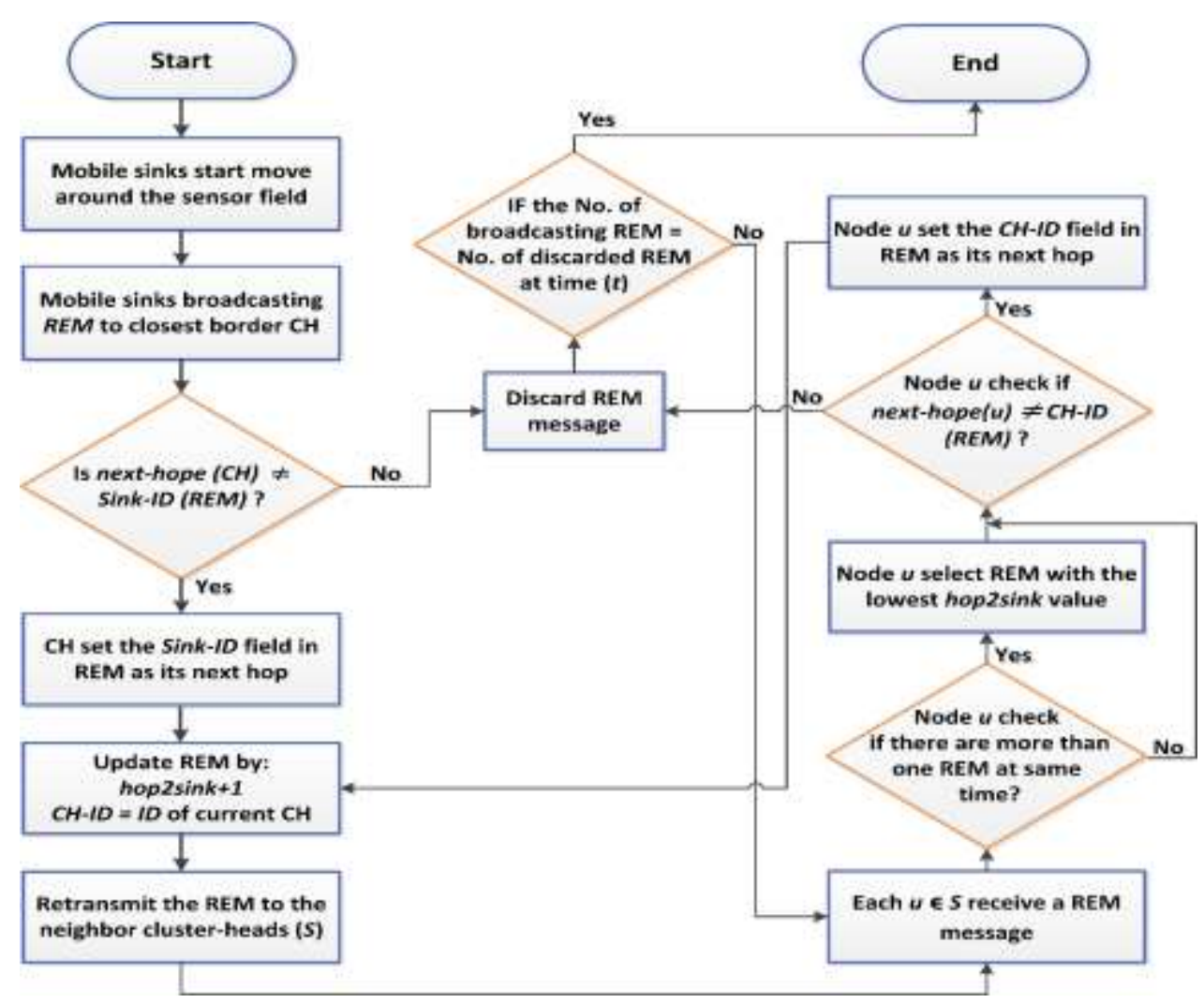

Figure 2. Flowchart of routes re-adjustment procedure 


\section{PERFORMANCE EVALUATION AND SIMULATION RESULTS}

For performance evaluation purposes, the suggested strategy (LRAS-MS) is compared with two other multi-mobile sink-based strategies, AVRP and DDRP. The simulations were carried out using MATLAB with IEEE 802.15.4 as the MAC layer protocol. The simulation environment was set up based on WSN in a periodically broadcast environment with an area of $(1000 \mathrm{~m} 2)$. In proposed strategy, a cluster structure is considered in the covered area and each sensor node belongs to cluster in the WSNs structure. In the system, two sinks collect data from the sensor nodes and travel around the monitoring zone uniformly as well as 600 source nodes. The velocity of the sinks varied from 0 to 20 , with a step increase of $4 \mathrm{~m} / \mathrm{s}$ while the field of the sensor, communication range and number of nodes were maintained.

Figure 3 provides a comparison of the energy consumption levels by calculating the energy consumption ratio of all nodes to the total number of network nodes. Results showed that LRAS-MS reduces energy consumption for various sink velocity by maintaining a lightweight route to the sinks when the sinks' positions are changed results in updating a small subset of nodes (as opposed to all nodes) in sensing field.

The lifetime of the network is highly influenced by the initial node energy and energy consumed over a specific period of time. As shown in Figure 4, the lifetime of all approaches in the network decreased as the velocity of the sinks increased in a fixed field size of the sensor. The superiority of LRAS-MS in terms of the network lifetime could be attributed to the energy-efficient mechanism adopted by LRAS-MS.

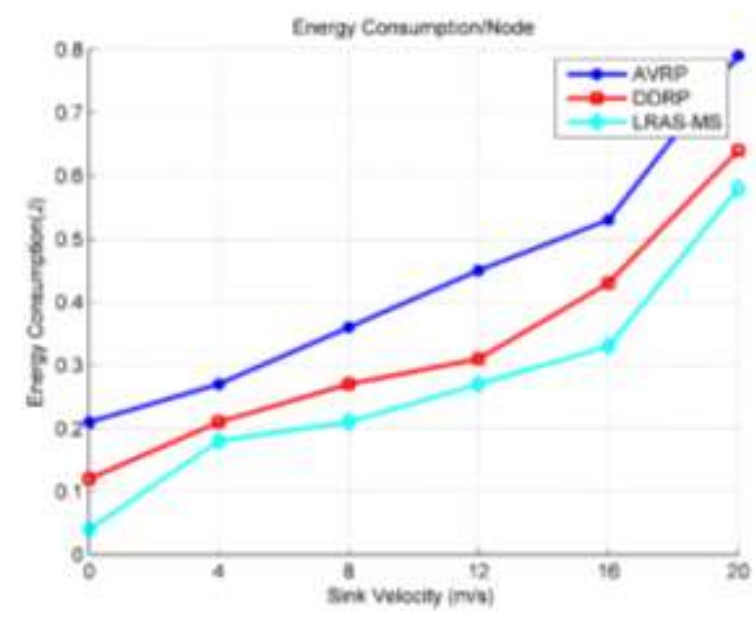

Figure 3. Comparison of energy consumption levels for different sink velocity

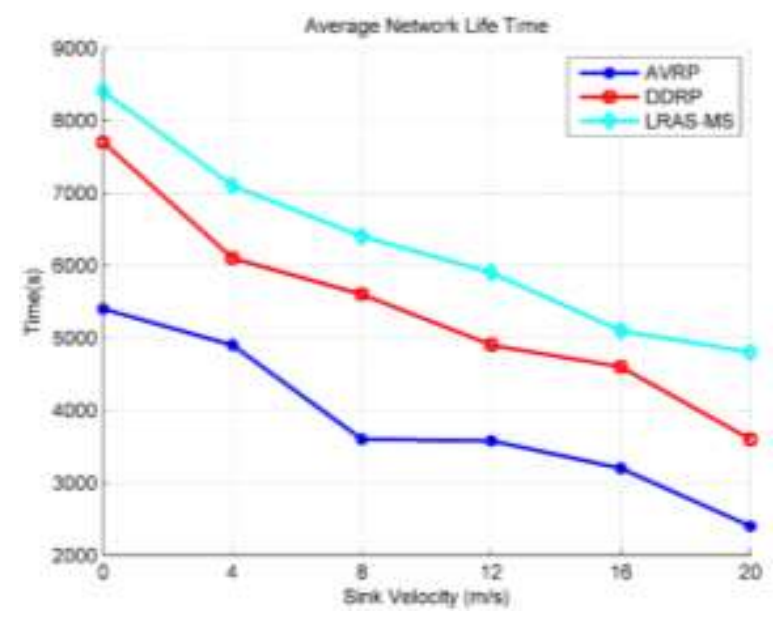

Figure 4. Comparison of the network lifetimes for different sink velocity

Figure 5 shows the LRAS-MS data delivery delay, which efficiently reduced the delay per delivered data and exceed both AVRP and DDRP in all tests in spite of the sink velocity. Due to sink mobility, the explanation for the direct relationship between delay and sink velocity triggers the rebuilding of a new path amidst the cluster heads and the mobile sink on the monitoring field whenever the sink varies the location.

Another important measurement metric is the data delivery ratio, used to measure the routing path efficiency developed by the tested approaches. As shown in Figure 6, at the lowest sinks' velocity, the data delivery ratio of LRAS-MS was 5.56\% and $11.76 \%$ respectively higher than those of AVRP and DDRP. While at the highest sinks' level, LRAS-MS' data delivery ratio was $53.97 \%$ and $21.25 \%$ respectively higher than AVRP and DDRP. This result is mainly due to the relay nodes in the routing path of the proposed strategy are less than those in the routing paths of AVRP and DDRP.

Finally, the control packet overhead of LRAS-MS and DDRP was typically comparable at a low sink velocity. As shown in Figure 7 , the minimum rates of control packet overhead $(0.11 \times 102-0.52 \times 102)$ were generated by LRAS-MS for different sink velocity. The average control packet overhead rates of the other two protocols, on the other hand, ranged from $(0.10 \times 102)$ to $(0.79 \times 102)$. This result is due to the only sub-set of cluster-head nodes involved in the reconstruction process of routes, thus minimizing overhead reconstruction overall routes while maintaining nearly optimal routes towards the current mobile sink spot. 


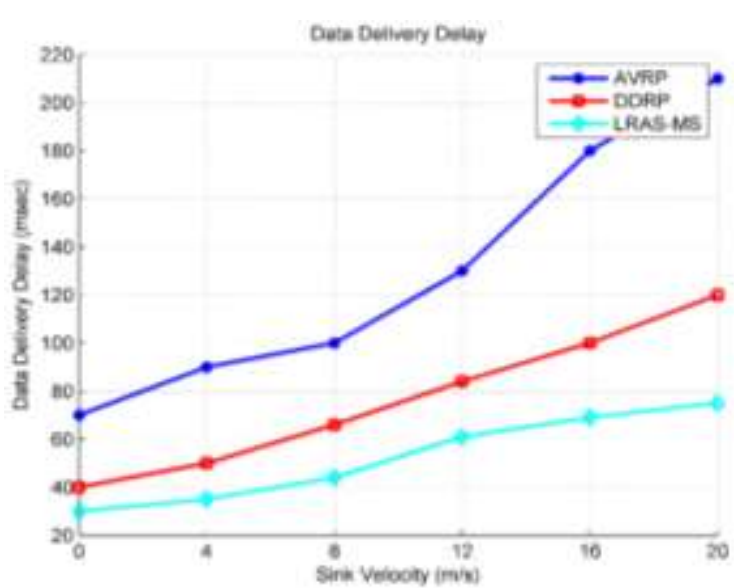

Figure 5. Comparison of the data delivery delay for different sink velocity

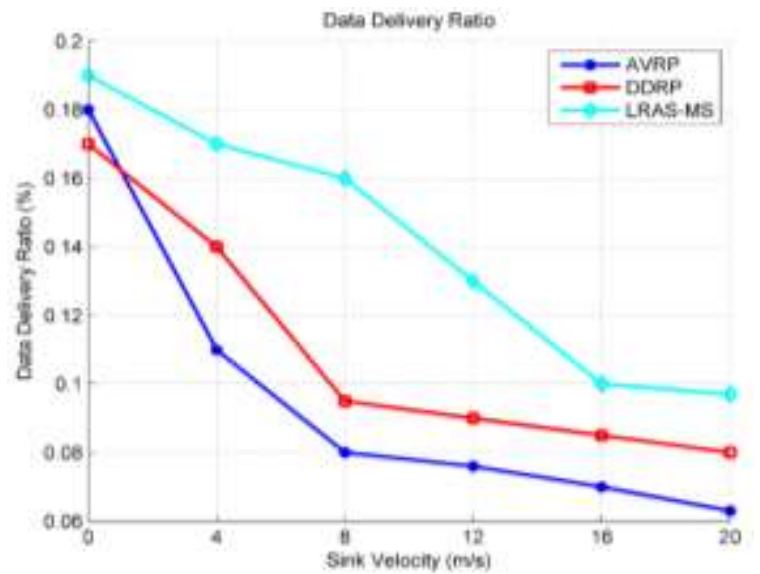

Figure 6. Comparison of the data delivery ratio for different sink velocity

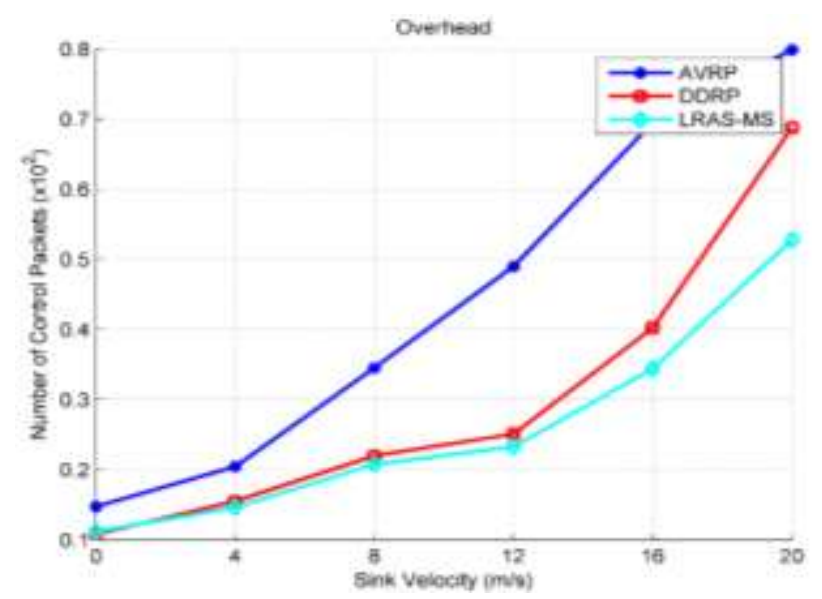

Figure 7. Overhead comparison for different sink velocity

\section{CONCLUSION}

Using mobile sinks to collect sensed information through the network is one common approach to alleviating the energy-hole problem and thus enhancing network existence. This paper presented the LRASMS strategy to make the best use of the advantage associated with sink mobility to minimize data communication. At the same time, this strategy will consider the cost of readjusting the routes in a multimobile WSN sink. The LRAS-MS can track the position of the mobile sink by considering only a sub-set of cluster-head nodes in the process of route re-adjustment. As for prospective works, it would be interesting to examine the LRAS-MS with the prediction of sink mobility and dynamic scheduling to adjust the route of the nodes according to the sink location learned from the algorithms of mobility prediction.

\section{REFERENCES}

[1] H. Alemdar and C. Ersoy, "Wireless sensor networks for healthcare: A survey," Computer networks, vol. 54, pp. 2688-2710, 2010.

[2] A. D. Salman, O. I. Khalaf, and G. M. Abdulsahib, "An adaptive intelligent alarm system for wireless sensor network," Indonesian Journal of Electrical Engineering and Computer Science (IJEECS), vol. 15, pp. 142-147, 2019.

[3] M. Alkhathami, L. Alazzawi, and A. Elkateeb, "Border surveillance and intrusion detection using wireless sensor networks," International Journal of Advances in Engineering \& Technology, vol. 8, p. 17, 2015.

[4] M. N. Ismail, M. A. Shukran, M. R. M. Isa, M. Adib, and O. Zakaria, "Establishing a soldier wireless sensor network (WSN) communication for military operation monitoring," Int. J. Inf. Commun. Technol,, vol. 7, pp. 89-95, 2018. 
[5] Y. R. B. Al-Mayouf, O. A. Mahdi, N. A. Taha, N. F. Abdullah, S. Khan, and M. Alam, "Accident management system based on vehicular network for an intelligent transportation system in urban environments," Journal of Advanced Transportation, vol. 2018, 2018.

[6] M. Y. I. Idris, A. W. A. Wahab, L. K. Qabajeh, and O. A. Mahdi, "Low communication cost (LCC) scheme for localizing mobile wireless sensor networks," Wireless Networks, vol. 23, pp. 737-747, 2017.

[7] O. A. Mahdi, A. W. A. Wahab, M. Y. I. Idris, A. A. Znaid, S. Khan, and Y. R. B. Al-Mayouf, "ESAM: endocrine inspired sensor activation mechanism for multi-target tracking in WSNs," in Fourth International Conference on Wireless and Optical Communications, p. 99020B, 2016.

[8] S. Yadav and R. S. Yadav, "A review on energy efficient protocols in wireless sensor networks," Wireless Networks, vol. 22, pp. 335-350, 2016.

[9] O. Adil Mahdi, A. W. Abdul Wahab, M. Y. I. Idris, A. Abu Znaid, Y. R. B. Al-Mayouf, and S. Khan, "WDARS: A weighted data aggregation routing strategy with minimum link cost in event-driven WSNs," Journal of Sensors, vol. 2016, 2016.

[10] S. Al-Khammasi, D. Alhelal, and N. S. Ali, "Energy Efficient Cluster Based Routing Protocol for Dynamic and Static Nodes in Wireless Sensor Network," TELKOMNIKA (Telecommunication, Computing, Electronics and Control), vol. 16, pp. 1974-1981, 2018.

[11] O. A. Mahdi, A. W. Abdul Wahab, M. Y. Idna Idris, A. M. Abu znaid, S. Khan, Y. R. B. Al-Mayouf, et al., "A comparison study on node clustering techniques used in target tracking WSNs for efficient data aggregation," Wireless Communications and Mobile Computing, vol. 16, pp. 2663-2676, 2016.

[12] D. Imededdin, A. Salih, and H. Medkour, "Design and implementation of low power consumption wireless sensor node," TELKOMNIKA (Telecommunication, Computing, Electronics and Control), vol. 17, pp. 2729-2734, 2019.

[13] M. H. Anisi, A. H. Abdullah, and S. A. Razak, "Energy-efficient and reliable data delivery in wireless sensor networks," Wireless Networks, vol. 19, pp. 495-505, 2013.

[14] A. T. ME, J. J. S. ME, A. K. Priya, R. Maarlin, and M. Harinetha, "Energy aware heuristic approach for cluster head selection in wireless sensor networks," Bulletin of Electrical Engineering and Informatics (BEEI), vol. 6, pp. 70-75, 2017.

[15] H. Barani, Y. Jaradat, H. Huang, Z. Li, and S. Misra, "Effect of sink location and redundancy on multi-sink wireless sensor networks: a capacity and delay analysis," IET Communications, vol. 12, pp. 941-947, 2018.

[16] A. Znaid, M. Ammar, M. Idris, Y. Idna, A. W. Abdul Wahab, L. Khamis Qabajeh, et al., "Sequential Monte Carlo localization methods in mobile wireless sensor networks: a review," Journal of Sensors, vol. 2017, 2017.

[17] W. Wen, C.-Y. Chang, S. Zhao, and C. Shang, "Cooperative Data Collection Mechanism Using Multiple Mobile Sinks in Wireless Sensor Networks," Sensors, vol. 18, p. 2627, 2018.

[18] M. Faheem and V. C. Gungor, "MQRP: Mobile sinks-based QoS-aware data gathering protocol for wireless sensor networks-based smart grid applications in the context of industry 4.0-based on internet of things," Future Generation Computer Systems, vol. 82, pp. 358-374, 2018.

[19] A. Khan, A. Abdullah, M. Anisi, and J. Bangash, "A comprehensive study of data collection schemes using mobile sinks in wireless sensor networks," Sensors, vol. 14, pp. 2510-2548, 2014.

[20] A. Kinalis, S. Nikoletseas, D. Patroumpa, and J. Rolim, "Biased sink mobility with adaptive stop times for low latency data collection in sensor networks," Information fusion, vol. 15, pp. 56-63, 2014.

[21] T. Banerjee, B. Xie, J. H. Jun, and D. P. Agrawal, "Increasing lifetime of wireless sensor networks using controllable mobile cluster heads," Wireless Communications and Mobile Computing, vol. 10, pp. 313-336, 2010.

[22] R. Mitra and S. Sharma, "Proactive data routing using controlled mobility of a mobile sink in Wireless Sensor Networks," Computers \& Electrical Engineering, vol. 70, pp. 21-36, 2018.

[23] A. M. Krishnan and P. G. Kumar, "An effective clustering approach with data aggregation using multiple mobile sinks for heterogeneous WSN," Wireless Personal Communications, vol. 90, pp. 423-434, 2016.

[24] K. Tian, B. Zhang, K. Huang, and J. Ma, "Data gathering protocols for wireless sensor networks with mobile sinks," in 2010 IEEE Global Telecommunications Conference GLOBECOM 2010, pp. 1-6, 2010.

[25] L. Shi, B. Zhang, H. T. Mouftah, and J. Ma, "DDRP: an efficient data-driven routing protocol for wireless sensor networks with mobile sinks," International Journal of Communication Systems, vol. 26, pp. 1341-1355, 2013.

[26] B. Tang, J. Wang, X. Geng, Y. Zheng, and J.-U. Kim, "A novel data retrieving mechanism in wireless sensor networks with path-limited mobile sink," Int. J. Grid Distrib. Comput, vol. 5, pp. 133-140, 2012.

[27] A. W. Khan, A. H. Abdullah, M. A. Razzaque, and J. I. Bangash, "VGDRA: a virtual grid-based dynamic routes adjustment scheme for mobile sink-based wireless sensor networks," IEEE Sensors Journal, vol. 15, pp. 526-534, 2014.

\section{BIOGRAPHIES OF AUTHORS}

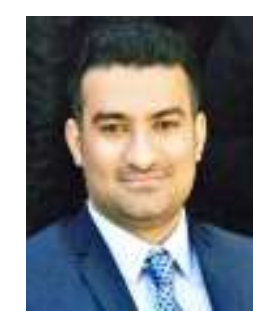

Ahmed B. Ghazi, received the M.Sc. degree in computer science from the faculty of computers and information, Helwan university, Egypt, in 2015. He currently works as a Teacher assistant at University of Baghdad, College of Education for Pure Science (Ibn al-Haitham), computer science department In Iraq. 


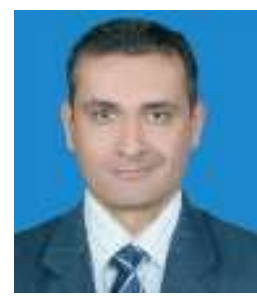

Omar Adil Mahdi was born in Baghdad, Iraq, in 1980. He received the bachelor's degree in computer science from University of Baghdad, Iraq, in 2003, the M.S. degree in computer science from University of Al-Anbar, Iraq, in 2010, and the Ph.D. degree from the Department of Computer System and Technology, Faculty of Computer Science and Information Technology, University of Malaya, Malaysia, in 2017. He is currently a lecturer in networks and communications with the Department of Computer Science, College of Education for Pure Science (Ibn Al-Haitham), University of Baghdad. His current research area is data aggregation and data routing in wireless sensor network, and wireless ad hoc, vehicular, sensor network, and next generation networks.

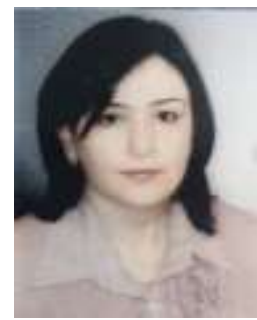

Wid Badee Abdulaziz She received the B.Sc. degree in systems and control engineering from University of Technology, Iraq, in 2002, and the M.Sc. degree in 2008. Currently, she is working toward the $\mathrm{PhD}$ at the same department. 